\title{
Invited Papers
}

\author{
2:40
}

4pAOa3. Integrated scheme of rapid environmental assessment for shallow water acoustics. Jean-Claude Le Gac (NATO Undersea Research Center, Viale San Bartolomeo 400, 19126 La Spezia, Italy, legac@nurc.nato.int), Jean-Pierre Hermand (Université libre de Bruxelles (U.L.B.) - Environmental hydroacoustics lab, av. Franklin D. Roosevelt 50, CP 194/5, 1050 Bruxelles, Belgium, jhermand@ulb.ac.be), Frans Absil (Royal Netherlands Naval College (NLDA) - REA group, PO Box 10000, 1780 Den Helder, Netherlands, fgj.absil@nlda.nl)

Predicting sound propagation in shallow or very shallow water environments requires that the frequency-dependent acoustic properties be assessed for all components of the waveguide, i.e., the water column, sea bottom and sea surface interface. During the Maritime Rapid Environmental Assessment MREA/BP'07 sea trial in April-May 2007, south of Elba Island in the Mediterranean Sea, an integrated MREA scheme has been implemented to provide a full 4D (3D+T) environmental picture that is directly exploitable by acoustic propagation models. Based on a joint multi-disciplinary effort, several standard and advanced techniques of environmental characterization covering the fields of underwater acoustics, physical oceanography and geophysics have been combined within a coherent scheme of data acquisition, processing and assimilation. The paper presents the whole architecture of the implemented scheme. Based on a preliminary analysis of MREA/BP'07 data, advantages and drawbacks of the approach will be discussed. Ways ahead for further improvement and perspectives are finally drawn.

\section{3:00}

4pAOa4. The application of rapid environmental assessment to sonar performance. Paul C. Hines (Defence R\&D Canada Atlantic, P.O. Box 1012, Dartmouth, NS B2Y3Z7, Canada, Paul.Hines@drdc-rddc.gc.ca), Sean Pecknold (Defence R\&D Canada Atlantic, P.O. Box 1012, Dartmouth, NS B2Y3Z7, Canada, sean.pecknold@drdc-rddc.gc.ca), John C. Osler (Defence R\&D Canada Atlantic, P.O. Box 1012, Dartmouth, NS B2Y3Z7, Canada, john.osler@drdc-rddc.gc.ca)

Naval sonar operations and planning in littoral environments requires information from historical databases, in situ sampling of environmental parameters, and models capable of estimating sonar performance and the uncertainty in the estimate. Defence R\&D Canada's approach to enable rapid environmental assessment (REA) for sonar incorporates three components: (1) a GIS-enabled database to manage historical environmental data, (2) measurement tools that operate while underway to provide in situ sampling of water column and seabed properties, and (3) a sensitivity model that examines the relative importance of different environmental parameters in order to quantify the impact of incomplete or degraded environmental information, and to specify the appropriate spatial and temporal scales for sampling. In this presentation, the integration of these aspects of REA will be demonstrated using transmission loss data collected in shallow water. Predictions made using REA data provide a substantially better fit to the measurements than those using historical databases.

\section{$3: 20$}

4pAOa5. Systemic approach to performance prediction and the exploitation of environmental information in naval systems. Patrick Grenard (DGA/DET/CTSN, BP28, 83800 Toulon Armées, France, Patrick.Grenard@dga.defense.gouv.fr)

Interoperability of systems and implementation of network enabled capabilities are at the heart of defense transformation. By providing extensive capabilities for data gathering, processing and presentations, they achieve a greater tactical advantage through superior knowledge of and use of the operational environment. Within the framework of the deployment of the new French Navy information system SIC21, a system of systems is being developed in order to support the implementation of the NATO concept of Recognized Environmental Picture (REP). The metasystem established allows for the assimilation of Rapid Environmental Assessment (REA) data, and for a local or remote production of the REP. In order to support decision making and to guide actions local exploitation of the REP is carried out through embedded tactical decision aids. They rely on advanced performance prediction tools including propagation modelling capabilities called in simulation scenarios. The aims of the implemented models are to predict performances at the appropriate level of granularity, to provide a confidence level on the proposed tactical picture, to define the appropriate level of marine environmental knowledge that should be acquired, to optimise sensor settings, and finally to provide inversion tools for the REA, ensuring consistency with forward modelling used in performance prediction. 